Boise State University

ScholarWorks

6-1-2016

\title{
Electron Beam Effects in Ge-Se Thin Films and Resistance Change Memory Devices
}

\author{
Kasandra Wolf \\ Boise State University \\ Hugh Barnaby \\ Arizona State University \\ Mahesh S. Ailavajhala \\ Boise State University \\ Micahel N. Kozicki \\ Arizona State University \\ Dmitri A. Tenne \\ Boise State University \\ See next page for additional authors
}


Authors

Kasandra Wolf, Hugh Barnaby, Mahesh S. Ailavajhala, Micahel N. Kozicki, Dmitri A. Tenne, and Maria Mitkova 
Wolf K, Ailavajhala MS, Tenne DA et al. (2016) Electron beam effects in Ge-Se thin films and resistance change memory devices. Emerging Materials Research 5(1): 126-134, http://dx.doi.org/10.1680/jemmr.15.00042
Emerging Materials Research Volume 5 Issue EMR 1

Themed issue: TMS 2015 - Symposium on advances in thin films for electronics and photonics Received 05/09/2015 Accepted 08/03/2016

Keywords: amorphous/composition/current voltage characteristics

\section{Electron beam effects in Ge-Se thin films and resistance change memory devices}

Kasandra Wolf MSC

Department of Electrical and Computer Engineering, Boise State University, Boise, ID, USA

Mahesh S. Ailavajhala PhD

Department of Electrical and Computer Engineering, Boise State University, Boise, ID, USA

Dmitri A. Tenne PhD

Department of Physics, Boise State University, Boise, ID, USA
Hugh Barnaby PhD

School of Electrical, Computer, and Energy Engineering, Arizona State University, Tempe, AZ, USA

Michael N. Kozicki PhD

School of Electrical, Computer, and Energy Engineering, Arizona State University, Tempe, AZ, USA

Maria Mitkova PhD*

Department of Electrical and Computer Engineering, Boise State University, Boise, ID, USA

Chalcogenide glasses are the advanced materials of choice for the emerging nanoionic memory devices - conductive bridge random access memory (CBRAM). To understand the nature of the effects occurring in these devices under influence of electron-beam radiation, the interaction of blanked chalcogenide films and nanostructured films containing chalcogenide glass and silver (Ag) source are studied. Raman spectroscopy, energy-dispersive X-ray spectroscopy and X-ray diffraction are used for establishing the structural and compositional effects occurring under radiation. They have strong compositional dependence with the stoichiometric compositions being most stable showing less structural changes after radiation. These effects are associated with the availability of lone-pair electrons, their participation in the bonding configurations and the coupling of electron states in the bandgap. They are further enhanced in the bilayers by silver diffusion in the chalcogenide matrix, as a result of interaction with electrons. These effects are used to interpret the electrical performance of CBRAM devices after radiation. The devices are characterized by their resistance states, threshold voltage and endurance. Those based on selenium-rich and stoichiometric composition undergo continuous parameters changes with increase in the radiation dose while in the devices based on germanium-rich composition a counter play of the structural changes and expulsion of silver occur.

\section{Introduction}

The rapid development of microelectronic systems has spurred the emergence of many new solutions to memory devices. One of the most promising of these is the programmable metallization cell or conductive bridge memory, which often because of the application these devices are mostly related with, is called conductive bridge random access memory (CBRAM). Their performance relies on the formation and dissolution of a conductive bridge between two electrodes. The bridge formation is driven by an oxireduction process during which ions from one electrode (anode), usually silver ions $\left(\mathrm{Ag}^{+}\right)$, move toward the other electrode (cathode), which is composed of an electrochemically inert material such as tungsten (W). At the interface of the tungsten electrode with the active device material (for example, chalcogenide glass), silver ions undergo reduction and convert to silver atoms. These silver atoms start coupling outward from the tungsten electrode, following the field distribution in the device and extending toward the silver electrode until a conductive bridge of silver atoms, connecting the two electrodes, forms. Applying an opposite bias between the electrodes dissolves this bridge. In this manner, the two conditions of the device are developed - an 'off' or high resistive state (HRS), in which there is no bridge between the two electrodes, and an 'on' or low resistive state (LRS), when the conductive bridge between the two electrodes is established. ${ }^{1}$ One of the best mediums in which the transport of ions could occur is based on chalcogenide glass film, particularly germanium $(\mathrm{Ge})$-selenium (Se) glass. Often this glass is diffused with silver, so as to encourage faster establishment of the conductive bridge. ${ }^{2}$ In order to demonstrate the wide range of applicability of these devices, it is important to study their performance in specific environments, particularly under the influence of electron beam (e-beam).

It is known that electrons cause material changes, for example the permittivity in the chalcogenide glasses, which inevitably will affect their electrical performance. ${ }^{3}$ The authors peculate that this is due to reorganization of the wrong bonds, but there are no structural data to prove this theory. In these glasses, the chemical order could be broken due to their non-equilibrium condition, especially in compositions containing high concentrations of germanium, in which there is departure from the N-8 coordination rule. ${ }^{4}$ The structure of these films, combining heteropolar Ge-Se 
bonding in tetrahedral organization with units of homopolar $\mathrm{Ge}-\mathrm{Ge}$ and Se-Se bonding, is particularly composition dependent. ${ }^{5,6}$

Other changes that the e-beam can cause in thin films of chalcogenide glasses are variations in the refractive index and film thickness, which the authors believe are caused by electroninduced surface contraction in the films. ${ }^{7}$ These results and the reported data about the formation of a relief over the surface of the chalcogenide films, under e-beam radiation, strongly suggest that the e-beam significantly affects the structure of the chalcogenide glasses inducing modifications in it. ${ }^{8-10}$ The nature of these modifications is currently not clear due to the lack of structural studies.

Another effect occurring in the chalcogenide glasses, under influence of electrons, is the impact of electrons on the silver distribution and migration within the chalcogenide glass. ${ }^{11,12}$ This effect is very important in the context of the CBRAM devices where silver plays a key role in their performance. In this case, it is imperative to know if silver migration, as a result of e-beam radiation, could be expected. The authors' hypothesis is that the structural reorganization mentioned earlier has a large influence on the diffusion of silver into the glass.

To complete the discussion about how an e-beam influences chalcogenide glasses, one has to also consider the charging effects it causes. Chalcogenide glasses are wide-bandgap semiconductors with bandgap ranging from $1 \cdot 7$ to $2 \cdot 5 \mathrm{eV},{ }^{13-15}$ and resistivity on the order of $10^{12} \Omega \mathrm{cm}^{15,16}$ These data qualify them rather as dielectric than semiconducting material, in which case, especially in structures with electrodes adjacent to the glass, charge will couple and redistribute, contributing to ion migration in the glass. $^{17}$

Having in mind the plurality of effects that could occur in the structure of the CBRAM devices, this work studied their performance after they have been irradiated with e-beam. In order to understand the nature of these effects, the structural changes occurring in the chalcogenide germanium-selenium films were studied, paying attention to the compositional dependence of the effects in both chalcogen-rich and chalcogen-poor films. Also studied were the products, forming as a result of the e-beam introduction of silver into the chalcogenide glasses, which provides insight into the understanding of the device performance.

\section{Experimental setup}

Bulk chalcogenide glasses of $\mathrm{Ge}_{x} \mathrm{Se}_{100-x}(x=20,30,40)$ were prepared from high-purity germanium and selenium by using the traditional melt-quench technique. The $\mathrm{Ge}_{x} \mathrm{Se}_{100-x}$ glass was then thermally deposited onto a silicon $(\mathrm{Si})$ wafer from a semi-Knudsen cell crucible in an attempt to create the $\mathrm{Ge}_{x} \mathrm{Se}_{100-x}$ bare films with composition close to that of the source material. Additional films were also produced where a silver source was selectively deposited onto the film surface to study the effect of e-beam-induced silver diffusion. Deposition of both $\mathrm{Ge}_{x} \mathrm{Se}_{100-x}$ and silver was achieved by using a Cressington 308R evaporation system at a pressure of $1 \times 10^{-6}$ mbar. The studied bare $\mathrm{Ge}_{x} \mathrm{Se}_{100-x}$ films were $75 \mathrm{~nm}$ thick. The $\mathrm{Ge}_{x} \mathrm{Se}_{100-x} /$ silver samples were assembled from $75 \mathrm{~nm}$ thick film of $\mathrm{Ge}_{x} \mathrm{Se}_{100-x}$ and $2 \mathrm{~mm}$-dia., $100 \mathrm{~nm}$-thick circular silver sources deposited on top of the chalcogenide films through a shadow mask. This structure was chosen since it allows the study of silver diffusion through its lateral distribution, which reduces the number of the variables for the result. If the diffusion in depth is studied, below the silver source, additional error would be introduced into the results.

The fabricated CBRAM devices were based on a silicon dioxide $\left(\mathrm{SiO}_{2}\right) /$ tungsten/ $\mathrm{Ge}_{x} \mathrm{Se}_{100-x} /$ silver vertical stack. The tungsten film was deposited by using an AJA Orion 5 sputter machine at a pressure of $6 \times 10^{-6} \mathrm{mbar}$. The thickness of each layer in the CBRAM devices was as follows: $200-\mathrm{nm}$ thermal silicon dioxide, 100 -nm tungsten, $75 \mathrm{~nm} \mathrm{Ge} \mathrm{Se}_{100-x}, 100 \mathrm{~nm}$ silver, in the device configuration shown in Figure 1.

Energy-dispersive spectroscopy (EDS), used to confirm the exact composition of each of the films, was conducted by using a Hitachi S-3400N II scanning electron microscope with an Oxford Instruments Energy + EDS system at a working distance of $10 \mathrm{~mm}$ and $\times 2000$ magnification with a $90 \mathrm{~s}$ collection time. Each sample was measured at five different locations for the collection of an accurate average and the standard deviation. Mapping profiles of diffused silver atoms, resulting from e-beam interactions, were acquired by using the same equipment, obtained from 20-25 averaging scans that rastered the beam to limit the exposure to electrons during analysis. The Raman spectra of bare films were collected by using a Horiba Jobin Yvon T64000 triple monochromator with a liquid-nitrogen-cooled, multichannel charge-coupled device detector. The films, inside a chamber at a temperature of $85 \mathrm{~K}$ and pressure of $1 \times 10^{-5} \mathrm{mbar}$, were excited by using a $441.6 \mathrm{~nm}$ helium (He)-cadmium (Cd) laser focused on an area with an $\sim 0.2 \mathrm{~mm}$ diameter and a laser intensity of $30 \mathrm{~mW}$. At this low temperature and vacuum, no photoinduced changes were established by sequential checks of the films by optical studies, although the laser light wavelength was within the absorption edge of the glasses.

The X-ray diffraction (XRD) patterns of silver diffused laterally outward from the silver dot sources were obtained by using a Bruker AXS D8 Discover X-ray diffractometer equipped with a thallium-doped sodium iodide $(\mathrm{NaI}(\mathrm{Tl}))$ scintillation detector and copper $(\mathrm{Cu}) \mathrm{K} \alpha \mathrm{X}$-ray source $(\lambda=0 \cdot 1506 \mathrm{~nm})$. The XRD

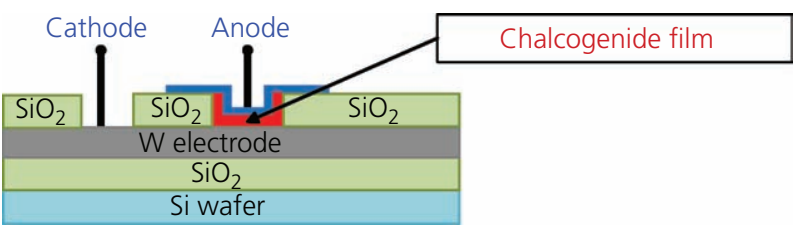

Figure 1. Cross-sectional view of W/Ge $\mathrm{Se}_{100-x} /$ silver CBRAM device 
Commander software was utilized in locked-coupled scan mode with $2 \theta$ ranging from $15^{\circ}$ to $90^{\circ}\left(0 \cdot 05^{\circ}\right.$ step size and $\left.1 \cdot 2 \mathrm{~s} / \mathrm{step}\right)$.

The $\mathrm{Ge}_{x} \mathrm{Se}_{100-x}$-based CBRAM devices were characterized by using an HP 4146 parameter analyzer. The probe station used for device characterization was equipped with gold probes and a Faraday cage, which isolated it from external sources of noise and diminished charge buildup within the measuring cables. The devices were characterized with a direct current voltage bias sweep, ranging from $-0 \cdot 75$ to $1 \cdot 5 \mathrm{~V}$ across the tungsten and silver electrodes, while simultaneously recording the current. In order to achieve $10^{5}$ switching cycles and more within a reasonable amount of time, a signal generator was utilized in addition to the parameter analyzer. The signal generator was programmed to supply a square waveform with a minimum of $-0.75 \mathrm{~V}$, a maximum of $2.0 \mathrm{~V}$ and a period of $5 \mathrm{~ms}$.

E-beam irradiation experiments were conducted by using a LEO $1430 \mathrm{VP}$ scanning electron microscope as an e-beam source with an accelerating voltage of $30 \mathrm{kV}$, beam current of $1 \mathrm{nA}$ and a working distance of $20 \mathrm{~mm}$. The beam diameter was $1.3 \mathrm{~mm}$, which resulted in an electron flux of $2.496 \times 10^{14}$ electrons/s. Bare films and such containing a silver source of the three different compositions were exposed to five separate doses of e-beam radiation $\left(0,2 \cdot 1 \times 10^{11}, 4 \cdot 2 \times 10^{11}, 6.3 \times 10^{11}\right.$ and $1.05 \times 10^{12}$ $\mathrm{rad})$ in order to provide a range of doses.

\section{Results}

The EDS study confirmed some differences in the composition of the studied films compared with the source material. The actual composition of the films also had some deviations from deposition to deposition of the same composition. On average, the samples contained germanium atomic concentrations of $x=$ $22 \cdot 6-25 \cdot 6,32 \cdot 4-36 \cdot 2$ and $42 \cdot 2-44 \cdot 4$, which in this study were termed further as selenium-rich, near-stoichiometric and germanium-rich films respectively.

The Raman spectra of control samples as evaporated and of samples that were subjected to the maximum dose of radiation are shown in Figure 2. They were normalized to the corner-sharing (CS) peak and fitted with Gaussian curves. The shape of the spectra demonstrates that the films are amorphous and they contain the characteristic structural units for the representative compositions. The Raman spectra of $\mathrm{Ge}_{25 \cdot 6} \mathrm{Se}_{74 \cdot 4}$ and $\mathrm{Ge}_{36 \cdot 2} \mathrm{Se}_{63 \cdot 8}$ films confirm the presence of three different structural units: CS tetrahedral structures $(\mathrm{Ge}-\mathrm{Se}-\mathrm{Ge})$ with average vibrational mode are present at $197 \mathrm{~cm}^{-1}$ and edge-sharing (ES) tetrahedral structures are present at $216 \mathrm{~cm}^{-1} ;^{18-21} \mathrm{Se}-\mathrm{Se}$ chains and rings are present at $270 \mathrm{~cm}^{-1,22,23}$ and the band that is observed at $308 \mathrm{~cm}^{-1}$ in the Raman spectra ${ }^{23-25}$ is attributed to the asymmetric average vibration of the edge-shared tetrahedron. The Raman spectra of the $\mathrm{Ge}_{36 \cdot 2} \mathrm{Se}_{63 \cdot 8}$ and the germanium-rich films (over 40 at.\% germanium) also indicate the presence of ethanelike bonding $(\mathrm{ETH})$ structures $\left(\mathrm{Se}_{3}-\mathrm{Ge}-\mathrm{Ge}-\mathrm{Se}_{3}\right)$ with an average vibrational mode at $178 \mathrm{~cm}^{-1}$.

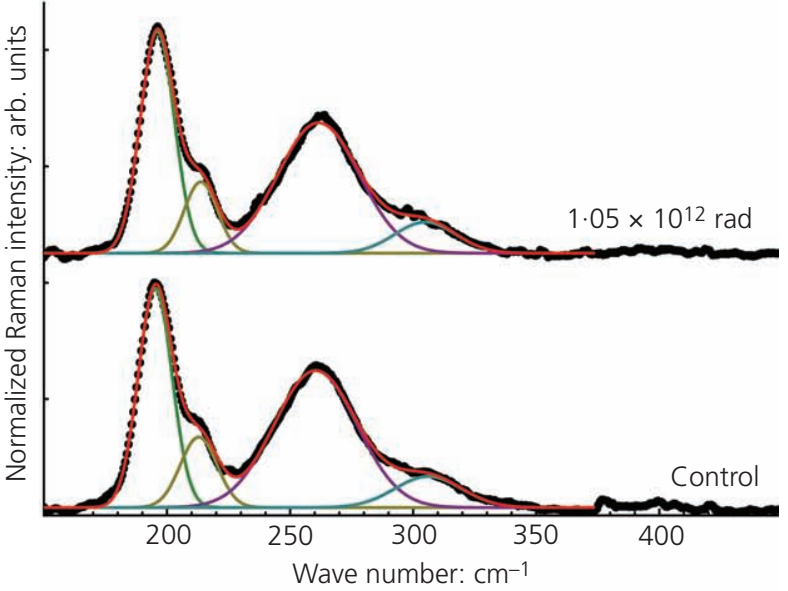

(a)

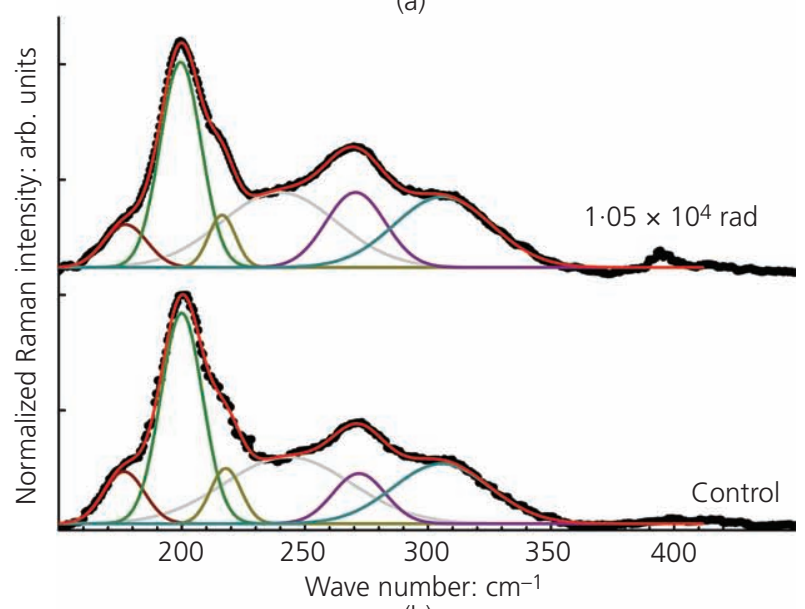

(b)

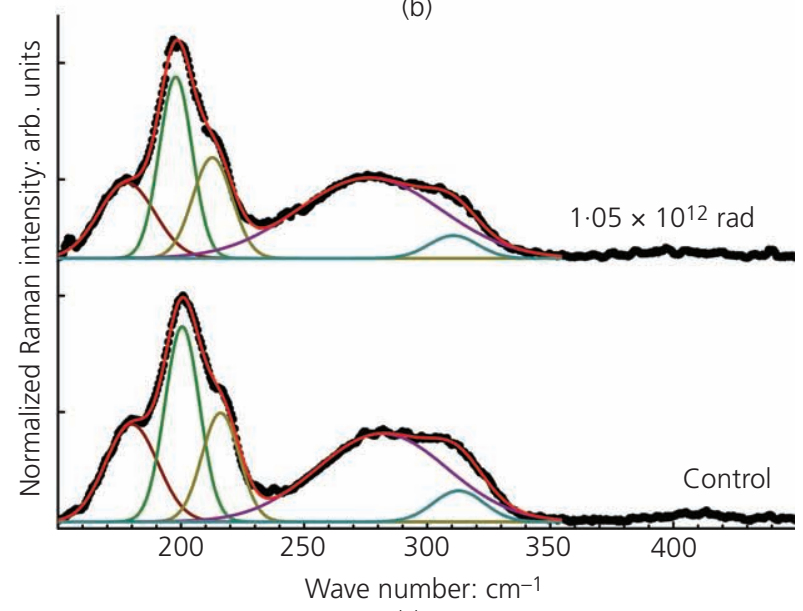

(c)

Figure 2. Raman spectra, normalized to the CS peak, of bare $\mathrm{a}-\mathrm{Ge}_{x} \mathrm{Se}_{100-x}$ films exposed to 0 and $1.05 \times 10^{12} \mathrm{rad}$ (highest dose) of e-beam for (a) $\mathrm{Ge}_{25 \cdot 6} \mathrm{Se}_{74 \cdot 4}$, (b) $\mathrm{Ge}_{36 \cdot 2} \mathrm{Se}_{63 \cdot 8}$ and (c) $\mathrm{Ge}_{44 \cdot 3} \mathrm{Se}_{55 \cdot 7}$

Analysis of the areal intensity of the various peaks reveals a unique trend as a function of e-beam exposure. The graph of the ratio of edge-sharing and corner-sharing tetrahedral structures (ES/CS), shown in Figure 3(a), provides evidence for relatively small structural changes in the development of the CS and ES 


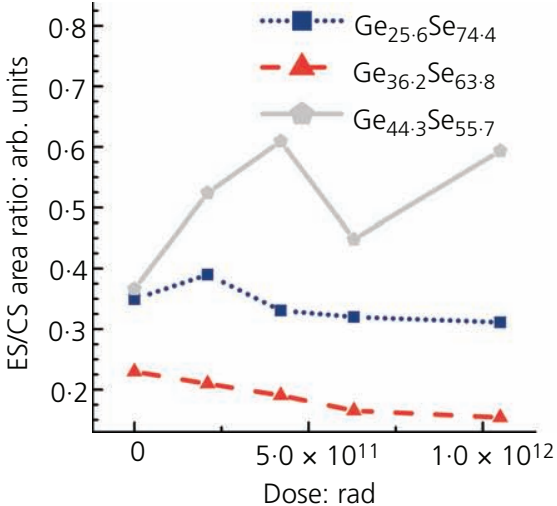

(a)

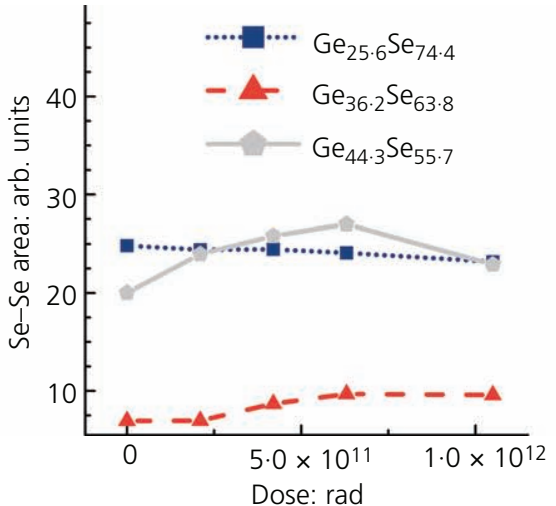

(b)

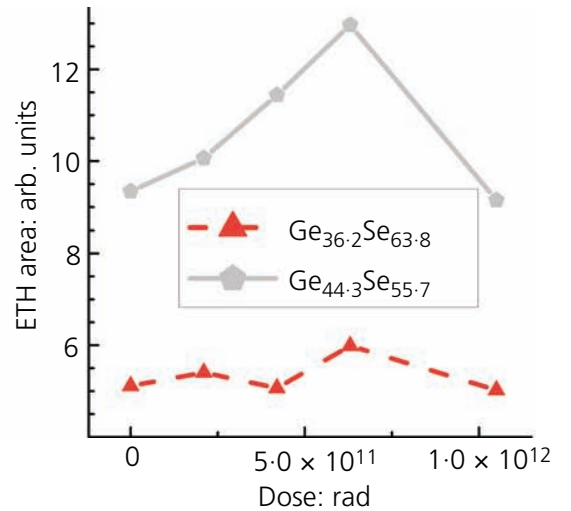

(c)
Figure 3. Changes in relative quantities of structural units, as extracted from Raman spectra, in a-Ge $\mathrm{Se}_{100-x}$ films ( $x=25 \cdot 6,36 \cdot 2$, 44.3) with $X$-ray exposure: (a) ratio of ES areal intensity to CS areal intensity (ES/CS); (b) areal intensity of Se-Se units; (c) areal intensity of ETH units structural units for selenium-rich samples and samples close to the stoichiometric composition, while the germanium-rich samples $(x=42-44$ at.\%) undergo structural reorganization with a local maximum at radiation of $5 \times 10^{11} \mathrm{rad}$ dose followed by a minimum and further reestablishment of the average ratio of the areal intensity of the ES/CS structural units. ${ }^{4,18,26}$ The development of the e-beam-induced effects in the Se-Se chains, shown in Figure 3(b), is also quite weak, with the biggest changes occurring for the germanium-rich samples. The ETH structural units are the most sensitive to irradiation. Their number increases in all germanium-rich samples at a radiation dose of up to $6.3 \times$ $10^{11} \mathrm{rad}$, after which an abrupt decrease in their areal intensity occurs (Figure 3(c)).

Raman studies on the silver-diffused germanium-selenium glasses were not carried out since such samples have a substantially lower intensity of their Raman modes. More detailed data about these samples were collected from XRD and EDS studies.

The XRD patterns of the e-beam-diffused silver in the area around the silver source are presented in Figures 4(a)-4(c). In each of the three compositions, $\alpha$-silver selenide $\left(\mathrm{Ag}_{2} \mathrm{Se}\right)$ and the ternary argyrodite $\left(\mathrm{Ag}_{8} \mathrm{GeSe}_{6}\right)$ form as a result of the reaction between the germanium-selenium glass film and the diffusing silver in it, supplied by the silver source (the dots on top of the chalcogenide films). The phase growth of both the $\alpha$-silver selenide and argyrodite nanocrystals in the chalcogenide matrix was observed, as a function of the radiation dose, which is illustrated in Figure 5. The $\alpha$-silver selenide phase was observed at $2 \theta=38^{\circ}$ angle, Joint Committee Powder Diffraction Standards (JCPDS) card 27-0619, in analogy with other cases in this system, ${ }^{27}$ and the argyrodite phase was observed at $2 \theta=44^{\circ}$, which is identified by JCPDS card 71-190. In addition to these two phases, a third phase of pure phase-separated silver is observed in $\mathrm{Ge}_{44.3} \mathrm{Se}_{55.7}$ at $2 \theta=61^{\circ}$, identified by JCPDS card 87-0598. Significant growth in the phase-separated silver was observed with increasing radiation dose. The distribution of the diffused silver atoms shows a proportional dependence on the electron energy as presented in Figure 5(b).

The films were also studied by using EDS mapping; the obtained result for $\mathrm{Ge}_{25.6} \mathrm{Se}_{74 \cdot 4}$ for the control sample and samples irradiated with $1.05 \times 10^{12} \mathrm{rad}$ is presented in Figures 6(a) and 6(b). When

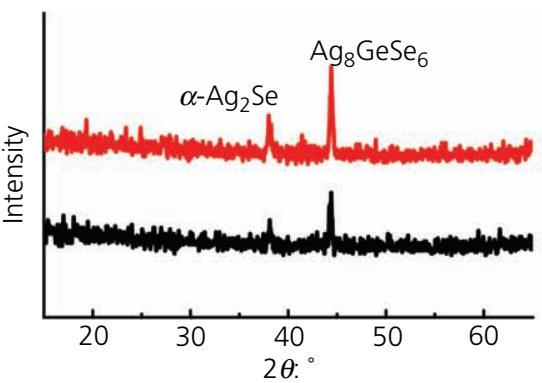

(a)

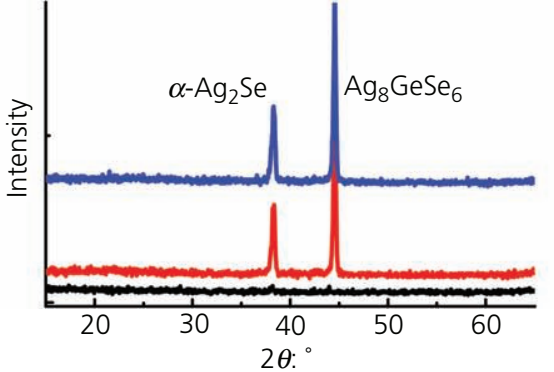

(b)

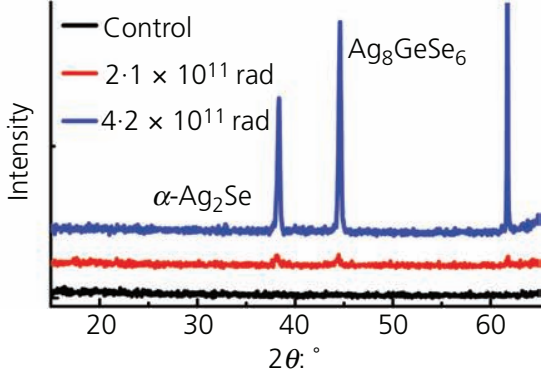

(c)

Figure 4. XRD patterns of silicon/silicon dioxide/ $\mathrm{Ge}_{x} \mathrm{Se}_{100-x} / \mathrm{silver}$ dot films exposed to $0,2.1 \times 10^{11}$ and $4.2 \times 10^{11}$ rad of e-beam with compositions (a) $\mathrm{Ge}_{25.6} \mathrm{Se}_{74.4}$, (b) $\mathrm{Ge}_{36.2} \mathrm{Se}_{63.8}$ and (c) $\mathrm{Ge}_{44.3} \mathrm{Se}_{55.7}$ 


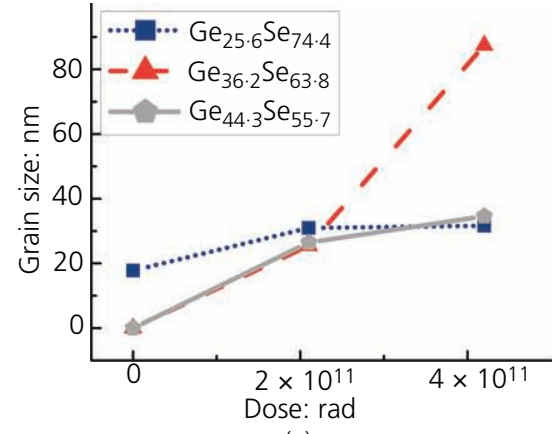

(a)

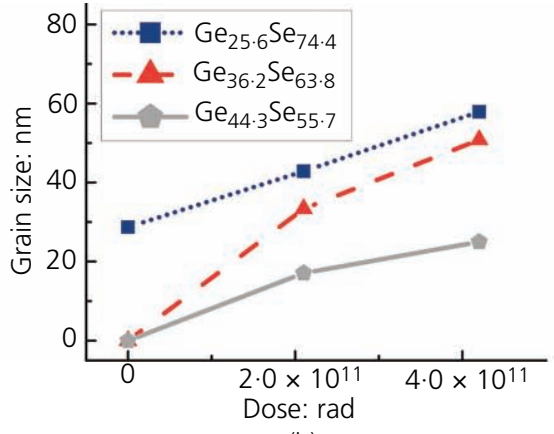

(b)

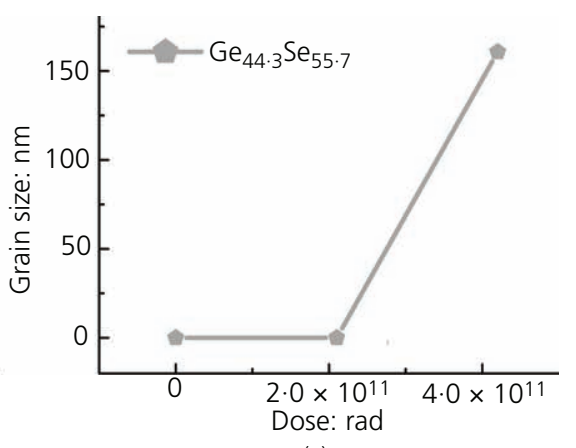

(c)

Figure 5. Grain size of germanium-selenium-silver species (a) silver selenide $\left(38^{\circ}\right)$, (b) argyrodite $\left(44^{\circ}\right)$ and (c) silver $\left(62^{\circ}\right)$ observed from XRD patterns

silver is detected by the detector, it is represented by a color depending on the intensity of the signal, while the lack of silver presence is represented by black regions on the image. The diameter of the silver source was measured and normalized to the diameter of the control sample, revealing the total diffusion distance of silver as a function of electron irradiation dose. Measured results illustrate a linear dependence between dose and diffusion distance. The diffusion distance is exaggerated in the selenium-rich films when compared with the other two compositions as shown in Figure 6(c).

CBRAM devices, based on three different compositions of $\mathrm{Ge}_{x} \mathrm{Se}_{100-x}(x=25 \cdot 6,36 \cdot 2$ and $44 \cdot 3)$, were tested for their current-voltage $(I-V)$ characteristics and endurance before and after e-beam exposure. Each device endured a minimum of $10^{5}$ switching cycles. The cumulative distributions of $V_{\mathrm{Th}}, V_{\mathrm{Er}}, \mathrm{LRS}$,

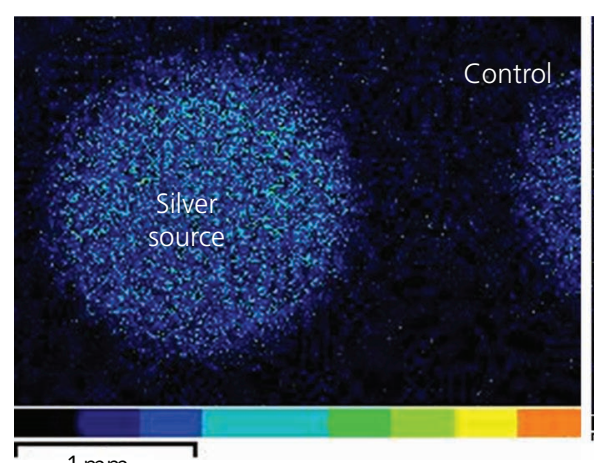

(a)

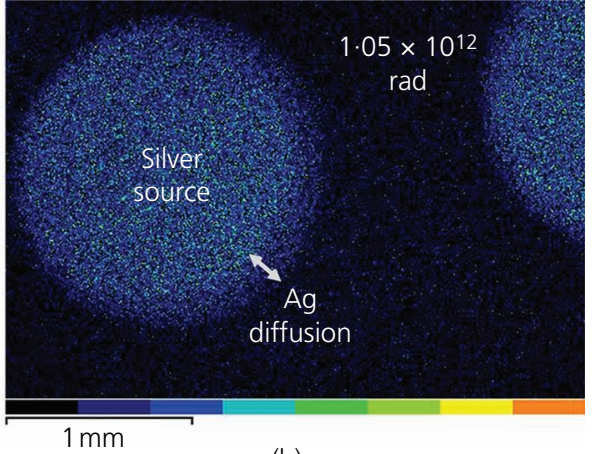

(b)

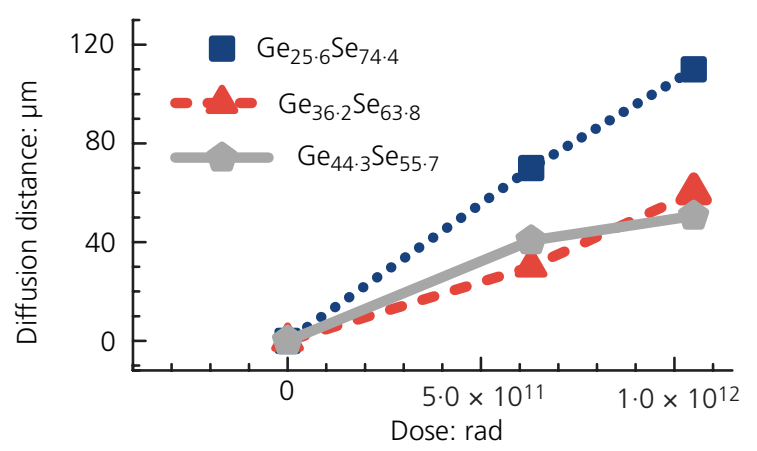

(c)

Figure 6. EDS mapping data of silver diffusion distribution in $\mathrm{Ge}_{25.6} \mathrm{Se}_{74.4}$ exposed to (a) 0 and (b) $1.05 \times 10^{12} \mathrm{rad}$; (c) total diffusion distance as determine from mapping data for three compositions 


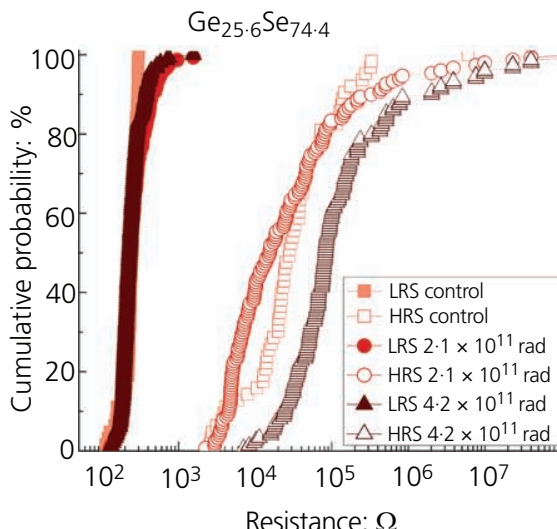

(a)

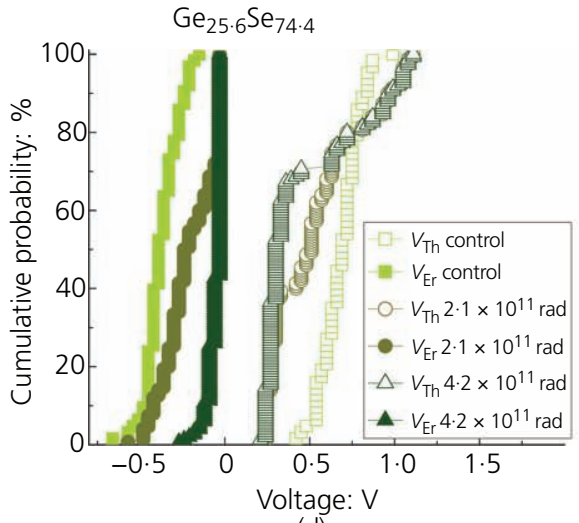

(d)

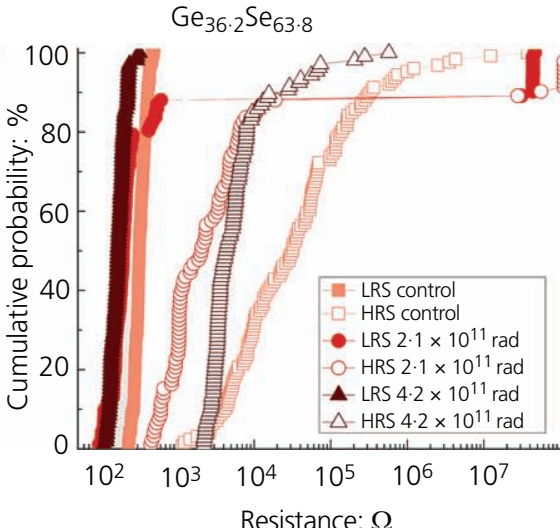

(b)

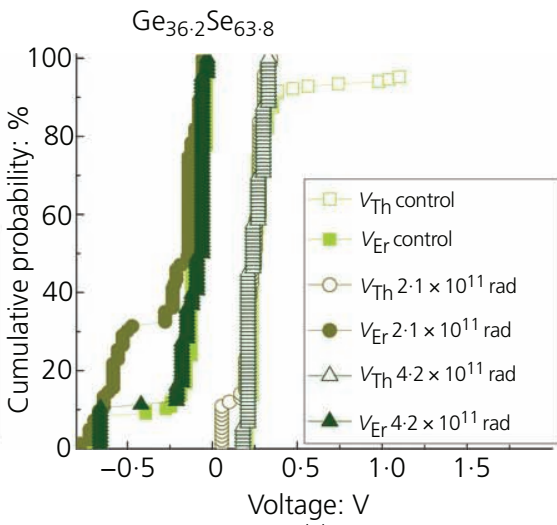

(e)

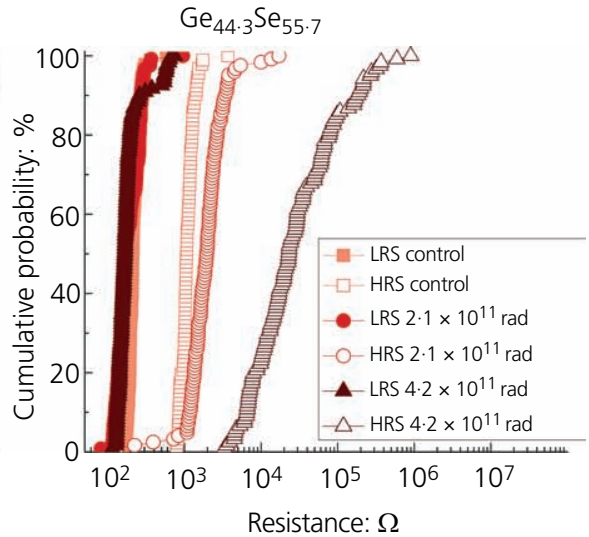

(c)

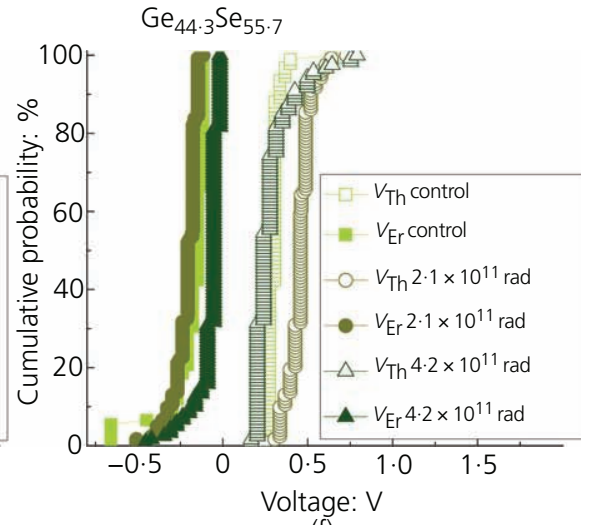

(f)
Figure 7. Distribution of (a-c) on/off-state resistances (red) and $(\mathrm{d}-\mathrm{f})$ write/erase voltages (green) for $\mathrm{Ge}_{x} \mathrm{Se}_{100-x}(x=25 \cdot 6,36 \cdot 2$, and 44.3) CBRAM devices exposed to $0,2.1 \times 10^{11}$ and $4.2 \times 10^{11} \mathrm{rad}$ of e-beam radiation: ( $a, d)$ selenium-rich; (b, e) near-stoichiometric; (c, f) germanium-rich and HRS for all compositions of devices are summarized in Figures 7(a)-7(c). I- $V$ curves are shown in Figure 7 for the selenium-rich, near-stoichiometric and germanium-rich devices exposed to three different doses of radiation. The cumulative distributions of the write/erase voltages for all compositions of devices are summarized in Figures 7(d)-7(f).

\section{Discussion}

The post-irradiation EDS data suggest that there are no losses of elements due to the interaction with the electrons. The electrons bombarding the sample penetrate into the film and undergo a number of random collisions by which they lose energy. The energy lost in the inelastic collisions is used for creation of electron-hole pairs. As a result of their formation, some of the bonds break, which further develops into structural changes. Both the selenium-rich and the near-stoichiometric compositions undergo relatively weak structural changes as a result of the electron bombardment. This can be associated with their quite floppy structure in which, after breakage of some bonding, fast relaxation occurs due to the structural flexibility. This fast recombination is facilitated by the intimate position of the selenium atoms in the selenium chains. ${ }^{28}$ Despite this, close evaluation of the areal intensities of the ES/CS ratio and $\mathrm{Se}-\mathrm{Se}$ structural units reveals that a transition occurs from ES (six selenium atoms) to CS (seven selenium atoms) structures, requiring more selenium, which is provided by the Se-Se chains. This is affirmed by the decrease in the intensity of the Se-Se Raman modes for the selenium-rich samples. In the case of the near-stoichiometric composition, as similarly in the case of the selenium-rich films, the intensity of the ES/CS ratio decreases while the Se-Se mode increases, which is also accompanied by an increase in the ETH structural units. In other words, in these structures, some atypical 'wrong bonds' (Se-Se and Ge-Ge bonds) appear as a result of the e-beam irradiation. The germanium-rich compositions undergo the most expressed structural changes, related to the low energy of the $\mathrm{Ge}-\mathrm{Ge}$ bonds available, which can easily break, and the fact that once a dangling bond occurs, it cannot immediately recombine due to the structural rigidity. The packing fraction of the germanium-rich glasses is very high, ${ }^{29}$ and this retards the diffusion of silver as shown in Figure 6(c). This is also the reason for the limited growth of silver clusters at low radiation doses (Figure 5(c)), which undergo fast growth after the structural changes associated with the destruction of the ETH structural units (Figure 3(c)) occur. 
The complexity of the effect of electron bombardment is further justified by the fact of formation of an internal steady state electric field due to the difference in the mobility of the electrons and holes. Its presence has been theoretically analyzed and discussed. ${ }^{30}$ This field contributes to an active motion within the solid and has been used for explanation of the processes of volume change in the chalcogenide glasses under the influence of e-beam. ${ }^{31}$ Under the influence of the field, some areas in the network experience electrostatic pressure, which contributes to the formation of voids and volume increase. It is for these two reasons - the field itself and the formation of less dense structure caused by it - that intense lateral diffusion of silver in the studied films has been observed.

Once diffused into the chalcogenide film, silver reacts with the network atoms. All of the silver containing $\mathrm{a}-\mathrm{Ge}_{x} \mathrm{Se}_{100-x}$ films revealed growth in both the binary $\alpha$-silver selenide and ternary argyrodite phases when exposed to e-beam radiation. However, the growth rate of the specific phases depends on the composition of the film as well as the exposure dose. It is important to note the preferential formation of the $\alpha$-silver selenide, which is stable only at temperatures above $133^{\circ} \mathrm{C}$, instead of $\beta$-silver selenide, which is stable at room temperature. The authors attribute the formation of $\alpha$-silver selenide in e-beam-exposed films, regardless of the composition, to the presence of high- and low-pressure regions resulting from radiation. The binary phase develops in these high-pressure regions, which forces the compound to form in its closest packed structure, face-centered cubic, rather than the orthorhombic structure ( $\beta$-silver selenide). The authors suggest that the main reasons for the longest diffusion pathway encountered for the selenium-rich samples are the high affinity between the silver and chalcogen elements, as well as the low packing of these films.

The mapping data demonstrate that at the conditions of the experiment, silver diffusion occurs on a distance much bigger than the thickness of the studied CBRAM devices. Consequently, one can expect that the device performance will be strongly affected by the silver diffusion. In the devices based on selenium-rich films, the median HRS decreases by a small amount for the lowest irradiation dose. After the first irradiation dose, proportional growth in both the $\alpha$-silver selenide and argyrodite phases are observed in the XRD patterns (Figures 5(a)) and 5(b)). The superconducting properties of the $\alpha$-silver selenide dominate the HRS value of the device after this first dose. The XRD patterns show that additional radiation causes further growth in the ternary phase; however, the binary phase is unchanged. Due to the further development in the ternary phase and the lack thereof in the binary phase, the semiconducting properties of the ternary phase govern the resistance of the device, resulting in a large increase in the HRS.

In devices based on material close to the stoichiometric composition, a large decrease in the HRS, after the first dose of irradiation, is revealed. This is attributed to the growth in both the $\alpha$-silver selenide and argyrodite phases as determined by the XRD patterns. After $10^{5}$ switching cycles, this device shows serious degradation in the device performance and device failure soon thereafter. Further e-beam irradiation results in the agglomeration of the $\alpha$-silver selenide phase crystal to form larger crystals spaced farther apart. The phase agglomeration, in addition to the spacing between these crystals, results in a higher HRS and decreasing variation in HRS due to fewer conductive paths for the bombarding electrons.

To summarize the results related to the devices based on selenium-rich and close to stoichiometric composition, the devices do not undergo very strong structural changes under electron radiation. However, because of they are abundant in selenium, the diffusion of silver is pretty much encouraged, which continuously changes the device parameters with increase in the dose of e-beam irradiation.

The HRS values of devices, based on germanium-rich films, demonstrate a large increase due to irradiation exposure, which the authors relate also to the observed large changes in the structure of the films. Virtually no change was observed in the HRS after the first dose of irradiation. There appears to be a threshold at irradiation of $4.2 \times 10^{11} \mathrm{rad}$, where the growth rates of the binary and ternary phases containing silver decreases. Additionally, the emergence of a phase-separated silver was observed from the XRD pattern. Furthermore, the ES/CS ratio increases dramatically (Figure 3(a)). The increase observed in the $\mathrm{ES} / \mathrm{CS}$ ratio results in the formation of voids and opening of the structural network. Consequently, incident electrons have a more direct path toward the previously diffused silver. Interaction between incident electrons and silver ionizes the silver atoms, making them more mobile. Additionally, charging at the interface between the silver electrode and the chalcogenide film creates an electric field. After $4.2 \times 10^{11} \mathrm{rad}$ of e-beam irradiation, the electric field becomes strong enough to withdraw silver ions from within the film. At the interface, silver agglomeration occurs, as shown by XRD patterns. This effect seems to be very similar to the one observed by Kawaguchi and Maruno ${ }^{32}$ for chalcogenide glasses containing silver. The changes culminate in a significant increase in the HRS after $4.2 \times 10^{11} \mathrm{rad}$. In this type of compositions, the device performance is governed by the counterplay of the structural changes in the chalcogenide matrix and expulsion of silver from it.

\section{Conclusions}

This study reveals that the e-beam irradiation affects the structure of Ge-Se films. The effect is further enhanced by silver diffusion into the chalcogenide films as a result of interaction with electrons. The electric field that forms during the bombardment of the Ge-Se films with electrons causes changes in the density of films and ion migration. This introduces silver across a much greater distance than the thickness of the studied CBRAM devices. As a result, their performance is affected by the presence of diffusion products such as silver selenide and argyrodite. Their amount and microcrystal growth are related to the structure of the 
accepting chalcogenide matrix. The devices based on seleniumrich and close to stoichiometric composition do not undergo very strong structural changes under electron radiation; but, because they are abundant in selenium, the diffusion of silver is pretty much boosted, which continuously changes the device parameters with increase in the dose of e-beam radiation. In the case of devices based on germanium-rich composition, their performance is governed by the counterplay of the structural changes in the chalcogenide matrix and expulsion of silver from it. These data could be used in material engineering for control of device parameters. The authors suggest that the stabilization of the performance of the CBRAM devices, under the influence of ebeam, can be achieved by saturation of the chalcogenide network with silver prior to the e-beam interaction - that is the technology of devices fabrication has to include a step for silver diffusion and saturation during device formation.

\section{Acknowledgements}

This work was funded by the Defense Threat Reduction Agency under Grant HDTRA1-11-1-0055.

\section{REFERENCES}

1. Kozicki MN, Park M and Mitkova M (2005) Nanoscale memory elements based on solid-state electrolytes. IEEE Transactions on Nanotechnology 4: 331-338.

2. Kozicki MN and Mitkova M (2006) Mass transport in chalcogenide electrolyte films - materials and applications. Journal of Non-Crystalline Solids 352: 567-577.

3. San-Román-Alerigi DP, Anjum DH, Zhang Y et al. (2013) Electron irradiation induced reduction of the permittivity in chalcogenide glass $\left(\mathrm{As}_{2} \mathrm{~S}_{3}\right)$ thin film. Journal of Applied Physics 113: 044116-044117.

4. Shatnawi MTM, Farrow CL, Chen $P$ et al. (2008) Search for a structural response to the intermediate phase in $\mathrm{Ge}_{x} \mathrm{Se}_{1-x}$ glasses. Physical Review B 77: 094134-11.

5. Boolchand P, Gunasekera K and Bhosle S (2012) Midgap states, Raman scattering, glass homogeneity, percolative rigidity and stress transitions in chalcogenides. Physica Status Solidi B 249: 2013-2018.

6. Darmawikarta K, Li T, Bishop SG and Abelson JR (2013) Two forms of nanoscale order in amorphous $\mathrm{Ge}_{x} \mathrm{Se}_{1-x}$ alloys. Applied Physics Letters 103: 131908-4.

7. Nordman O, Nordman N and Peyghambarian N (1998) Electron beam induced changes in the refractive index and film thickness of amorphous $\mathrm{As}_{x} \mathrm{~S}_{100-x}$ and $\mathrm{As}_{x} \mathrm{Se}_{100-x}$ films. Journal of Applied Physics 84: 6055-6058.

8. Tanaka K (1997) Electron beam induced reliefs in chalcogenide glasses. Applied Physics Letters 70: 261-263.

9. Romero JS, Fitzgerald AG and Mietzsch K (2002) Electron irradiation induced expansion in amorphous chalcogenide films. Journal of Applied Physics 91: 9572-9574.

10. Debnath RK and Fitzgerald AG (2005) Electron beam induced surface modification of amorphous $\mathrm{Sb}_{2} \mathrm{~S}_{3}$ chalcogenide films. Applied Surface Science 243: $148-150$
11. Liang YC and Tada K (1988) Silver distribution in wetsensitized $\mathrm{As}_{10} \mathrm{Ge}_{22 \cdot 5} \mathrm{Se}_{67 \cdot 5}$ films after electron beam exposure. Journal of Applied Physics 64: 1378-1383.

12. Yoshida N and Tanaka K (1997) Ag migration in Ag-As-S glasses induced by electron-beam irradiation. Journal of NonCrystalline Solids 210: 119-129.

13. Smektala F, Quemard C, Couderc V and Barthélémy A (2000) Non-linear optical properties of chalcogenide glasses measured by Z-scan. Journal of Non-Crystalline Solids 274: 232-237.

14. Pan RK, Tao HZ, Wang JZ et al. (2013) Structure and optical properties of amorphous Ge-Se films prepared by pulsed laser deposition. Optik - International Journal for Light and Electron Optics 124: 4943-4946.

15. Kawaguchi T, Maruno S and Elliott SR (1996) Optical, electrical, and structural properties of amorphous Ag-Ge-S and Ag-Ge-Se films and comparison of photoinduced and thermally induced phenomena of both systems. Journal of Applied Physics 79: 9096-9104.

16. Tronc $P$, Bensoussan M, Brenac A and Sebenne C (1973) Optical-absorption edge and Raman scattering in $\mathrm{Ge}_{x} \mathrm{Se}_{1-x}$ glasses. Physical Review B 8: 5947.

17. Melchinger A and Hofmann S (1995) Dynamic double layer model: description of time dependent charging phenomena in insulators under electron beam irradiation. Journal of Applied Physics 78: 6224-6232.

18. Sugai S (1987) Stochastic random network model in Ge and Si chalcogenide glasses. Physical Review B 35: 1345-1361.

19. Inoue K, Matsuda O and Murase K (1996) A model calculation of the characteristic Raman modes in the tetrahedral network structures of $\mathrm{GeSe}_{2}$. Physica B: Condensed Matter 219-220: 520-522.

20. Jackson K (2000) Electric fields in electronic structure calculations: electric polarizabilities and IR and Raman spectra from first principles. Physica Status Solidi (b) 217: 293-310

21. Edwards TG and Sen S (2011) Structure and relaxation in germanium selenide glasses and supercooled liquids: a Raman spectroscopic study. The Journal of Physical Chemistry B 115: 4307-4314.

22. Lucovsky G, Wong CK and Pollard WB (1983) Vibrational properties of glasses: intermediate range order. Journal of Non-Crystalline Solids 59-60: 839-846.

23. Lucovsky G and Galeener FL (1980) Intermediate range order in amorphous solids. Journal of Non-Crystalline Solids 35-36: 1209-1214.

24. Kumar RR, Barik A, Vinod E et al. (2013) Crossover from photodarkening to photobleaching in $\mathrm{a}-\mathrm{Ge}_{x} \mathrm{Se}_{100-x}$ thin films. Optics Letters 38: 1682-1684.

25. Jackson K, Briley A, Grossman S, Porezag D and Pederson M (1999) Raman-active modes of a-GeSe $\mathrm{G}_{2}$ and a-GeS $\mathrm{G}_{2}$ : a firstprinciples study. Physical Review B 60: R14985-R14989.

26. Bridenbaugh PM, Espinosa GP, Griffiths JE, Phillips JC and Remeika JP (1979) Microscopic origin of the companion $A_{1}$ Raman line in glassy $\mathrm{Ge}(\mathrm{S}, \mathrm{Se})_{2}$. Physical Review $B$ 20: 4140-4144. 
27. Mitkova M, Kozicki M, Kim H and Alford T (2006) Crystallization effects in annealed thin Ge-Se films photodiffused with Ag. Journal of Non-Crystalline Solids 352: 1986-1990.

28. Barik AR, Bapna M, Drabold DA and Adarsh KV (2014) Ultrafast light induced unusually broad transient absorption in the sub-bandgap region of $\mathrm{GeSe}_{2}$ thin film. Science Reports 4: 3686, http://dx.doi.org/10.1038/srep03686.

29. Chen P, Mitkova M, Tenne DA et al. (2012) Study of the sorption properties of $\mathrm{Ge}_{20} \mathrm{Se}_{80}$ thin films for $\mathrm{NO}_{2}$ gas sensing. Thin Solid Films 525: 141-147.
30. Thomas C, Joahimsthaler I, Heiderhof and Balk LJ (2004) Electron-beam-induced potentials in semiconductors: calculation and measurement with an SEM/SPM hybrid system. Journal of Physics D: Applied Physics 37: 2785-2794.

31. Cserhati C, Charnovych S, Lytvyn PM et al. (2012) E-beam induced mass transport in amorphous $\mathrm{As}_{20} \mathrm{Se}_{80}$ films. Materials Letters 85: 113-116.

32. Kawaguchi T and Maruno S (1995) Photoinduced surface deposition of metallic silver in Ag-As-S glasses. Journal of Applied Physics 77: 628-634.

\footnotetext{
WHAT DO YOU THINK?

To discuss this paper, please submit up to 500 words to the managing editor at journals@ice.org.uk.

Your contribution will be forwarded to the author(s) for a reply and, if considered appropriate by the editorin-chief, will be published as a discussion in a future issue of the journal.

ICE Science journals rely entirely on contributions sent in by professionals, academics and students coming from the field of materials science and engineering. Articles should be within 5000-7000 words long (short communications and opinion articles should be within 2000 words long), with adequate illustrations and references. To access our author guidelines and how to submit your paper, please refer to the journal website at www.icevirtuallibrary.com/emr
} 